\title{
Genetic signature of differential sensitivity to stevioside in the Italian population
}

\author{
Davide Risso • Gabriella Morini • Luca Pagani • \\ Andrea Quagliariello • Cristina Giuliani - Sara De Fanti • \\ Marco Sazzini · Donata Luiselli · Sergio Tofanelli
}

Received: 15 November 2013/Accepted: 25 March 2014/Published online: 6 April 2014

(C) Springer-Verlag Berlin Heidelberg 2014

\begin{abstract}
The demand for diet products is continuously increasing, together with that for natural food ingredients. Stevioside and other steviol glycosides extracted from the leaves of the plant Stevia rebaudiana Bertoni are the first natural high-potency sweeteners to be approved for consumption in the United States and the European Union. However, the sweetness of these compounds is generally accompanied by aversive sensations, such as bitter and offtastes, which may constitute a limit to their consumption. Moreover, consumers' differences in sensitivity to highpotency sweeteners are well known, as well as difficulties in characterizing their aftertaste. Recently, TAS2R4 and TAS2R14 have been identified as the receptors that mediate the bitter off-taste of steviol glycosides in vitro. In
\end{abstract}

Donata Luiselli and Sergio Tofanelli are co-senior authors.

Electronic supplementary material The online version of this article (doi:10.1007/s12263-014-0401-y) contains supplementary material, which is available to authorized users.

D. Risso $(\varangle) \cdot$ L. Pagani · A. Quagliariello · C. Giuliani ·

S. De Fanti - M. Sazzini · D. Luiselli

Laboratory of Molecular Anthropology and Centre for Genome

Biology, Department of BiGeA, University of Bologna, via

Selmi 3, 40126 Bologna, Italy

e-mail: davide.risso2@unibo.it

\section{G. Morini}

University of Gastronomic Sciences, Piazza Vittorio Emanuele

9, Pollenzo, 12042 Bra, Cn, Italy

L. Pagani

The Wellcome Trust Sanger Institute, Hinxton CB10 1SA, UK

S. Tofanelli

Department of Biology, University of Pisa, Via Ghini 13,

56126 Pisa, Italy the present study, we demonstrate that TAS2R4 gene polymorphism rs2234001 and TAS2R14 gene polymorphism rs3741843 are functional for stevioside bitterness perception.

Keywords Stevioside $\cdot$ Bitter aftertaste $\cdot$ Genetic polymorphisms · hTAS2R4 - hTAS2R14 · hTAS2R38

\section{Introduction}

Stevioside (Fig. 1) is the most abundant compound of a group of structurally related high-potency sweeteners, the steviol glycosides, which are secondary metabolites extracted from the leaves of the Stevia rebaudiana Bertoni. This plant is native to Paraguay where its leaves have a long history of use as sweetener and to treat several diseases (Kinghorn 2002; Yadav and Guleria 2012; VegaGálvez et al. 2012). All these compounds share a common aglycone, known as steviol (ent-13-hydroxykaur-16-en-18oic acid), but differ in the number and types of sugar residues.

All steviol glycosides are high-potency sweeteners, and stevioside has been reported to have a relative sweetness, compared to sucrose, between 210 and 300, depending on the protocol used (Crammer and Ikan 1987; Kinghorn and Soejarto 1986). A detailed study of concentration/response functions for several sweeteners, including stevioside, was presented by DuBois et al. (1991). The demand for this kind of sweeteners in the production of zero and reducedcalorie food products is continuously expanding as a response to increasing health awareness. Such awareness stems from a growing body of evidence showing that overweight and obesity strongly contribute to a large proportion of non-communicable diseases. 


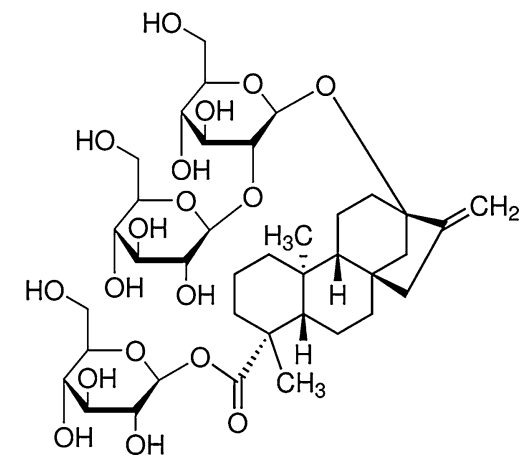

Fig. 1 Chemical structure of stevioside

As for other high-potency sweeteners (DuBois and Prakash 2012), the sweet taste of steviol glycosides presents bitter and off-tastes (Kinghorn and Soejarto 1986; Prakash et al. 2008). Moreover, consumers' differences in sweeteners sensitivity and acceptance, as well as the difficulties in characterizing their aftertaste, have been reported along with evidences suggesting that most of variation in sensitivity to chemical stimuli may have a genetic basis (Simons et al. 2008).

The recent introduction (2008 in USA, 2011 in EU) of purified steviol glycosides (at least $95 \%$ ) as the first natural high-potency sweeteners on the market opens up questions on the genetic basis of stevioside taste perception and its worldwide distribution. Particularly, understanding the differential ability to sense stevioside bitter aftertaste of various human populations has implications on its worldwide use as a natural sweetener and, consequently, on the diffusion of related healthy reduced-calorie food products. Bitter taste is detected by a set of 25 taste 2 receptors (TAS2R) (Meyerhof et al. 2010), and individual differences in the ability to taste substances like phenylthiocarbamide have been known since a long time (Fox 1932). Single nucleotide polymorphisms (SNPs) in the TAS2R38 gene have been identified as the key determinants of this capability, as well as for that of tasting the related compound 6- $n$-propylthiouracil (PROP) (Bufe et al. 2005; Kim et al. 2003; Duffy et al. 2004). In particular, PROP status (i.e., taster or non-taster) has been proposed by some authors to have a broad effect on other oral sensations and therefore has been considered a marker for food preferences and diet selection (Tepper 2008). However, this issue is highly debated as other studies showed that PROP responsiveness had no influence on food preferences (Baranowski et al. 2011).

Recently, hTAS2R4 and hTAS2R14 have been identified as the receptors that specifically mediate the bitter offtaste of steviol glycosides in vitro (Hellfritsch et al. 2012), opening the way to investigate whether variation at the genes encoding for these receptors could explain individual differences in aftertaste perception.
To date, only some SNPs in chemosensory genes have been actually associated with differential perception of bitter compounds (Allen et al. 2013a; Campbell et al. 2013; Roudnitzky et al. 2011; Reed et al. 2010; Wooding et al. 2010; Pronin et al. 2007). Nevertheless, genetic differences in sensitivity to natural bitter compounds are an aspect of great relevance considering that bitter taste receptors are expressed also in the gastrointestinal (GI) tract (and in other extra-oral tissues). In fact, the GI tract represents the key interface between food and the human body and bitter taste receptors have been proven to play a crucial role in the control of several functions, ranging from gastric emptying to appetite modulation, to the detection of ingested harmful compounds and toxins, to emesis (Reimann et al. 2012).

That being so, in this work, we set to investigate putative associations between stevioside bitter aftertaste and SNPs on genes known to be involved in bitterness perception (i.e., TAS2R4, TAS2R14 and TAS2R38), in a panel of Italian subjects. Furthermore, we explore their worldwide patterns of diversity, looking for signatures of ongoing selection at these loci that could explain potential differences in the ability to sense stevioside bitter aftertaste in different human populations.

\section{Subjects and methods}

Study design and sampled population

A total of 86 healthy adult Italian donors (48 females and 38 males) were recruited. Subjects (average age of $47 \pm 14$ years) were not following a prescribed diet or using drugs that might interfere with taste perception, and none of them had food allergies. In addition, individuals who smoked more than ten cigarettes per week were excluded.

\section{Experimental protocol}

A training session regarding taste perception, taste qualities and how to rate perception using the Labeled Magnitude Scale (LMS, Green et al. 1993) was performed. Volunteers were asked to refrain from eating and drinking for at least 3 $\mathrm{h}$ before the beginning of the session and to rinse their mouth with room temperature deionized water prior to the first and between each sample. Weight (in $\mathrm{kg}$ ) and height (in $\mathrm{m}$ ) were collected, in order to calculate the body mass index (BMI). For the perception test, $10 \mathrm{~mL}$ of a solution of stevioside $1.26 \mathrm{mM}$ (Nastevia, Stevia Italia s.r.l., Italy) was used. The PROP-taster status was assessed using cotton swabs dipped in $50 \mathrm{mM}$ 6-n-propylthiouracil solution (Sigma Aldrich S.r.l.) (Caremoli 2011), modifying the 
Table 1 Details of the studied SNPs in TAS2R4, TAS2R14 and TAS2R38 genes

\begin{tabular}{|c|c|c|c|c|c|c|}
\hline Chr & Gene & dbSNP & cSNP & Allele & Type of mutation & Residue change \\
\hline \multirow[t]{2}{*}{7} & \multirow[t]{2}{*}{ TAS2R4 } & \multirow[t]{2}{*}{ rs2234001 } & \multirow[t]{2}{*}{ G286-C } & G & \multirow[t]{2}{*}{ Missense } & $\mathrm{V}$ [Val] \\
\hline & & & & $\mathrm{C}$ & & L [Leu] \\
\hline \multirow[t]{6}{*}{12} & \multirow[t]{6}{*}{ TAS2R14 } & \multirow[t]{2}{*}{ rs11610105 } & \multirow[t]{2}{*}{ G11088981-A } & G & \multirow[t]{2}{*}{ Silent } & \multirow[t]{2}{*}{1} \\
\hline & & & & A & & \\
\hline & & \multirow[t]{2}{*}{ rs3741843 } & \multirow[t]{2}{*}{ G375-A } & G & \multirow[t]{2}{*}{ Cds synon } & $\mathrm{R}$ [Arg] \\
\hline & & & & A & & $\mathrm{R}$ [Arg] \\
\hline & & \multirow[t]{2}{*}{ rs7138535 } & \multirow[t]{2}{*}{ A114-T } & A & \multirow[t]{2}{*}{ Cds synon } & G [Gly] \\
\hline & & & & $\mathrm{T}$ & & G [Gly] \\
\hline \multirow[t]{6}{*}{7} & \multirow[t]{6}{*}{ TAS2R38 } & \multirow[t]{2}{*}{ rs713598 } & \multirow[t]{2}{*}{ G229-C } & G & \multirow[t]{2}{*}{ Missense } & A [Ala] \\
\hline & & & & $\mathrm{C}$ & & P [Pro] \\
\hline & & \multirow[t]{2}{*}{ rs10246939 } & \multirow[t]{2}{*}{ A970-G } & A & \multirow[t]{2}{*}{ Missense } & I [Ile] \\
\hline & & & & G & & $\mathrm{V}$ [Val] \\
\hline & & \multirow[t]{2}{*}{ rs1726866 } & \multirow[t]{2}{*}{ T869-C } & $\mathrm{T}$ & \multirow[t]{2}{*}{ Missense } & V [Val] \\
\hline & & & & $\mathrm{C}$ & & A [Ala] \\
\hline
\end{tabular}

protocol for filter paper discs described in Zhao et al. (2003). Subjects were informed that they may receive stimuli eliciting more than one taste quality. They were asked to held the stevioside solution in the mouth for $5 \mathrm{~s}$ and the cotton PROP swab for 10 s. For both stimuli (i.e., stevioside and PROP), participants were allowed to choose among different tastes such as bitter, sweet or tasteless, as well as to rate their perceived intensity separately on multiple LMSs, one for each taste quality.

DNA collection, extraction, genotyping and quality control

DNA was collected using Oragene saliva collection kits and extracted according to the manufacturer's protocol (Genotek Inc., Kanata, Ontario, Canada). A list of genotyped SNPs in TAS2R14 (chromosome 12), TAS2R4 and TAS2R38 (chromosome 7), which were selected from the literature and by surveying lists of genetic variants contained in different databases, is shown in Table 1. Genotypes for these variants were determined using Sequenom MassARRAY technology (Sequenom, San Diego, CA, USA). Genotype calls were analyzed by using SEQUENOM Typer 4.0 software, and the individual spectrograms were checked in order to evaluate the presence of calling errors. None of them showed significant deviations from Hardy-Weinberg equilibrium (Online Resource 1). We also assessed their minor allele frequency to be above $5 \%$ and their call rate to be above $95 \%$.

Statistical analyses

Allelic and genotypic association analyses were performed with PLINK v. 1.07 (Purcell et al. 2007) using Fisher's exact method, and the significance threshold was adjusted according to the Bonferroni multiple testing correction (adjusted $P=P$ value $\times$ number of individual tests). Linkage disequilibrium (LD) patterns at the TAS $2 R 38$ gene were explored by means of the solid spine approach implemented in the Haploview package (Barrett et al. 2005). The PHASE software (Stephens et al. 2001) was then used to statistically infer TAS2R38 haplotypes within the identified high-LD blocks and to reconstruct haplotype pairs for each subject. For association analyses between individual SNPs and bitterness perception, analysis of covariance (ANCOVA) was performed using STATISTICA v. 6.0 (Stat-Soft Inc, Tulsa, OK), considering age, sex and BMI as covariates. Analysis of molecular variance (AMOVA) was carried out with Arlequin v. 3.5 (Excoffier et al. 2007). Exploration of worldwide variation patterns for the examined SNPs was performed using 1000 Genomes Project PHASE 1, HapMap and Human Genome Diversity Project datasets. Functional annotations and SIFT (http://sift.jcvi.org/) predictions (Kumar et al. 2009) for each SNP were retrieved from the Ensembl database (http://ensembl.org).

\section{Results}

Assessing taste phenotypes

Volunteers involved in the study were classified, after the perception test, in different tasting categories for both PROP and stevioside. Regarding the PROP status, the distribution of PROP sensitivity showed the classical bimodal curve (Online Resource 2), with 18 individuals $(20.9 \%)$ being classified as non-tasters and $68(79.1 \%)$ as 


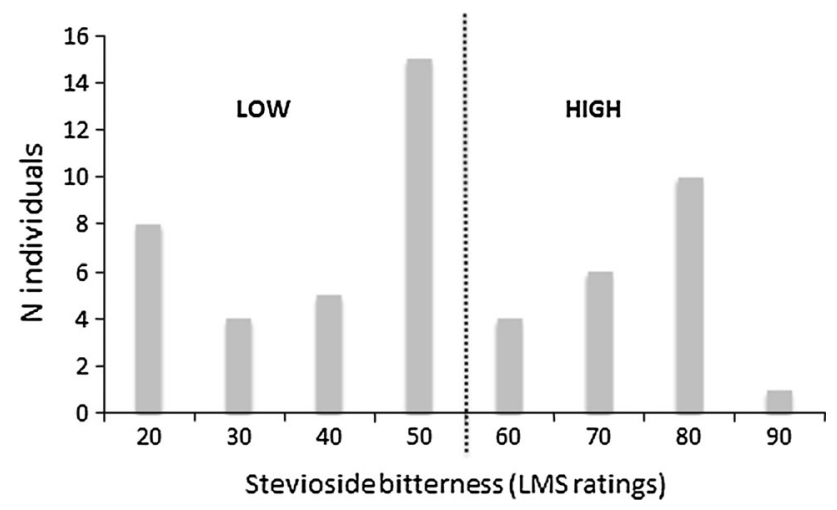

Fig. 2 Distribution of stevioside sensitivity in the Italian sample

tasters. In order to test whether the adopted deviation from the method described in Zhao et al. (2003) could affect PROP phenotype assessment, we calculated the overall genotype-phenotype concordance $(90.7 \%)$, thus inferring the correctness of our cotton swab PROP test. After stevioside tasting, 11 individuals ( $13 \%$ ) were able to perceive only its bitter taste. On the contrary, 22 individuals ( $26 \%$ ) perceived only its sweet taste, whereas the majority of samples constituted by 53 individuals $(61 \%)$ identified a sweet taste followed by a bitter after-taste.

The $53 \mathrm{bitter} / \mathrm{sweet}$-tasters were subsequently distinguished into 32 "bitter-low" (LMS scores up to 50) and 21 "bitter-high" (LMS from 60 to 100) tasters (Fig. 2) according to LMS scale scores.

TAS2R4 SNP regulates the ability to perceive the bitter taste of stevioside

The TAS2R4 rs2234001 (C/G) turned out to be associated with stevioside bitter status. In fact, the "bitter" and "sweet" phenotype groups showed statistically significant differences for both genotypic (adjusted $P=0.002$, Fisher's exact test) and allelic frequencies (adjusted $P=0.039$, Fisher's exact test). In particular, the GG genotype and the $\mathrm{G}$ allele were more frequent in bitter-tasters $(n=11)$, whereas genotype $\mathrm{CC}$ and the $\mathrm{C}$ allele were more frequent in sweet-tasters $(n=22)$. More specifically, $68.18 \%$ of bitter-tasters carried the $\mathrm{G}$ allele, whereas $76.19 \%$ of sweet-tasters carried the $\mathrm{C}$ allele at this locus (Table 2). The same segregation was observed between the sweet and the "bitter-low" tasters $(n=32)$, with the genotype GG (adjusted $P=0.008$, Fisher's exact test) and the $G$ (adjusted $P=0.013$, Fisher's exact test) allele being more frequent in the "bitter-low" group. Analyses conducted with the SIFT software showed that rs2234001 caused an amino acidic substitution at residue 96 , resulting in a valine-leucine change, without altering the secondary structure of the protein. However, this SNP was found to be
Table 2 Distribution of TAS2R4 rs2234001 and TAS2R14 rs 3741843 polymorphisms among stevioside phenotypes

\begin{tabular}{|c|c|c|c|}
\hline rs2234001 & Bitter & Bitter/sweet & Sweet \\
\hline Genotype & $\%$ & $\%$ & $\%$ \\
\hline $\mathrm{CC}$ & 0 & 19.22 & 57.1 \\
\hline $\mathrm{CG}$ & 63.64 & 50 & 38.1 \\
\hline GG & 36.36 & 30.78 & 4.8 \\
\hline \multicolumn{4}{|l|}{ Allele } \\
\hline $\mathrm{C}$ & 31.82 & 44.23 & 76.19 \\
\hline G & 68.18 & 55.77 & 23.81 \\
\hline rs 3741843 & \multicolumn{2}{|c|}{ Bitter-low } & Bitter-high \\
\hline Genotype & \multicolumn{2}{|c|}{$\%$} & $\%$ \\
\hline AA & \multicolumn{2}{|c|}{93.55} & 42.85 \\
\hline GA & \multicolumn{2}{|c|}{6.45} & 57.15 \\
\hline GG & \multicolumn{2}{|c|}{0} & 0 \\
\hline \multicolumn{4}{|l|}{ Allele } \\
\hline A & \multicolumn{2}{|c|}{96.77} & 28.57 \\
\hline $\mathrm{G}$ & \multicolumn{2}{|c|}{3.23} & 71.43 \\
\hline
\end{tabular}

in strong LD $\left(r^{2} \geq 0.9\right)$ in European populations with two other non-synonymous SNPs (rs2227264 and rs2233998), both predicted by SIFT to alter the function of TAS2R5 and TAS2R4 proteins, respectively.

TAS2R4 SNP does not predict variations in stevioside bitterness perception

We also tested whether TAS2R4 rs2234001 (C/G) could predict stevioside bitterness or sweetness. Differently than TAS2R14 rs3741843, this SNP did not show variation among different levels of bitter and sweet perception (ANCOVA, $P=0.601$ and $P=0.623$, respectively).

TAS2R14 SNP predicts variations in stevioside bitterness perception

We found evidence that TAS2R14 rs3741843 (A/G) has a significant impact on bitterness perception. Through comparing the "bitter-low" and "bitter-high" groups, the allelic frequency of this SNP was found to be significantly different (adjusted $P=0.002$; Fisher's exact test). In particular, the $\mathrm{G}$ allele was more frequent in the "bitter-high" group $(n=21)$ compared to the "bitter-low" one $(n=32)$. Genotypic analyses confirmed this statistically significant different distribution, with genotype AA being more frequent in the "bitter-low" group (adjusted $P \leq 0.001$, Fisher's exact test). The same tests were repeated by removing the LMS modal group $(\mathrm{LMS}=50$ ), which is suspected to introduce a confounding effect, and obtained results confirmed our previous estimation 
Fig. 3 Effect of the TAS2R14 rs3741843 on bitterness and sweetness of stevioside and on bitterness of PROP

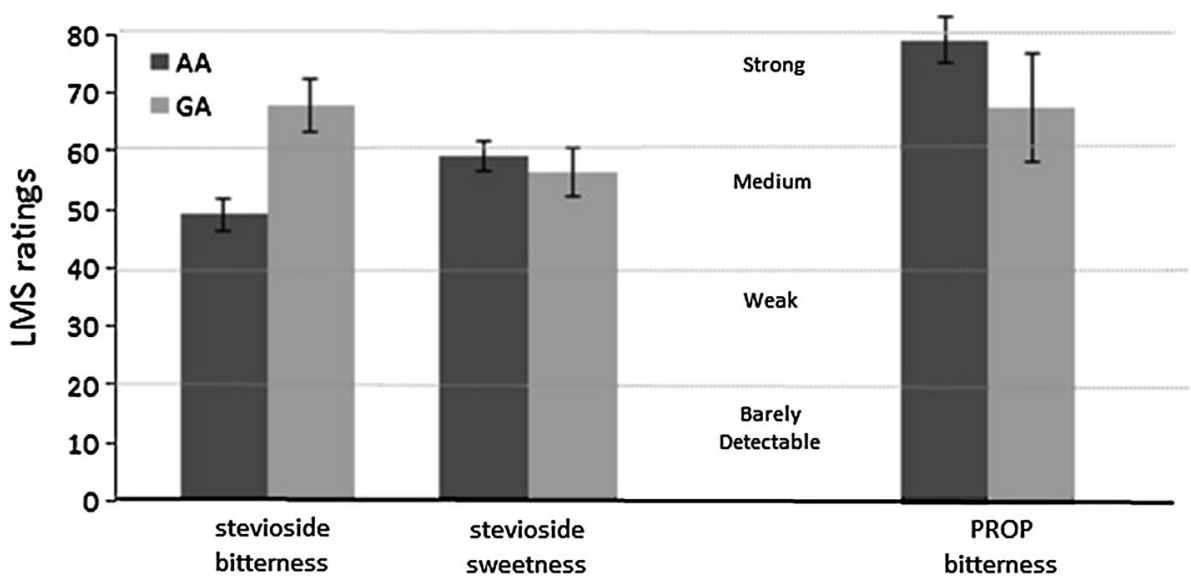

$(P=0.003$ and $P=0.001, \quad$ Fisher's exact test, respectively).

In addition, to test whether arbitrary classifications in taste phenotypes were plausible, an ANCOVA was performed on the subset of individuals who were able to perceive stevioside bitterness $(n=64)$, considering age, sex and BMI as covariates. As shown in Fig. 3, homozygote individuals with the AA genotype of TAS2R14 rs3741843 reported less bitterness from stevioside than heterozygote ones $(P=0.002)$. In contrast, there was no evidence that this allele predicts stevioside sweetness (ANCOVA, $P=0.621$ ). We also found minimal evidence that stevioside bitterness is predictive of PROP bitterness (ANCOVA $P=0.081$ ).

TAS2R38 SNPs predict variations in PROP bitterness but not in stevioside bitterness perception

As expected, PROP-tasters and non-tasters differed significantly for TAS2R38 alleles and haplotypes. In particular, TAS2R38 rs10246939 (T/C), rs1726866 (T/C) and rs713598 (G/C) were more frequent in PROP-tasters ( $n=68)$ compared to PROP-non-tasters $(n=18)$ (adjusted $P<0.001$, adjusted $P<0.001$, adjusted $P<0.001$, Fisher's exact tests). In the same way, perception of PROP bitterness varied with TAS2R38 haplotypes. The prolinealanine-valine (PAV) homozygotes $(n=15)$ reported significantly more bitterness than the heterozygotes ( $n=42$ ) (ANCOVA, $P<0.001$ ) or the AVI (alanine, valine, and isoleucine) homozygotes $(n=24)$ (ANCOVA, $P<0.001)$. Moreover, bitterness reported by heterozygotes was similar to that reported by PAV homozygotes (ANCOVA, $P=0.345$ ). Rare haplotypes were excluded from the analyses because they are known to have intermediate phenotypes that differ from both each other and the common haplotypes. In this study, we observed both AAI (3) and AAI/AVI (2) individuals. No associations were instead found between PROP haplotypes and stevioside bitterness (ANCOVA, $P=0.945$ ) or sweetness (ANCOVA, $P=0.812$ ).

Comparison between stevioside bitterness and sweetness perception

Great variability in both stevioside bitterness and sweetness perception was found. Figure 4 shows this distribution of variation, with stevioside bitterness and sweetness ranging from 20 to 80 on a LMS. We therefore tried to test whether a covariation between them existed. By plotting the bitterness and sweetness of stevioside simultaneously, we did not observe a covariation between them $\left(R^{2}=0.007, \quad P=0.776\right)$, with bitterness showing a decreasing trend toward the modal class and sweetness a decreasing trend followed by an increase after the modal class.

Population genetics of TAS2R4 rs2234001 and TAS2R14 rs3741843 SNPs

\section{Alleles worldwide distribution}

To better understand the differences observed for TAS $2 R 4$ and TAS2R14 SNPs, we examined their worldwide distribution. Figure 5 shows a map of allele frequencies of the two studied SNPs, based on 1000 Genomes Project data. Hardy-Weinberg test (Online Resource 3) and Heterozygosity test (Online Resource 4) were performed, confirming that all the examined populations are in HardyWeinberg equilibrium and no excess of heterozygosity was found $(P>0.05)$.

\section{AMOVA}

In order to identify the causes underlying the different distributions of rs3741843 and rs2234001, AMOVA based on the 1000 Genomes Project data was performed. As 
Fig. 4 Scatter plot showing sweetness and bitterness intensities of stevioside. Histograms shown along the axes illustrate the total amount of variation found across individuals
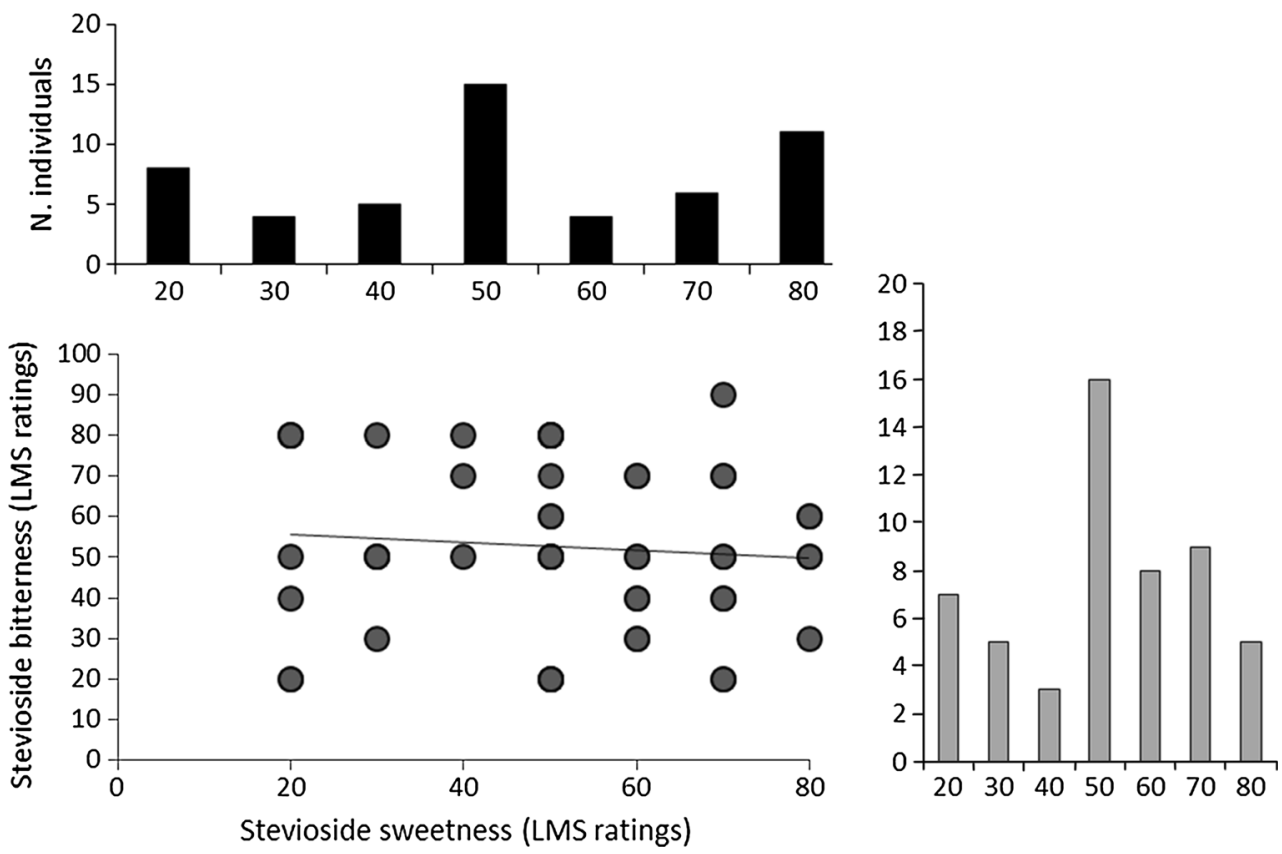

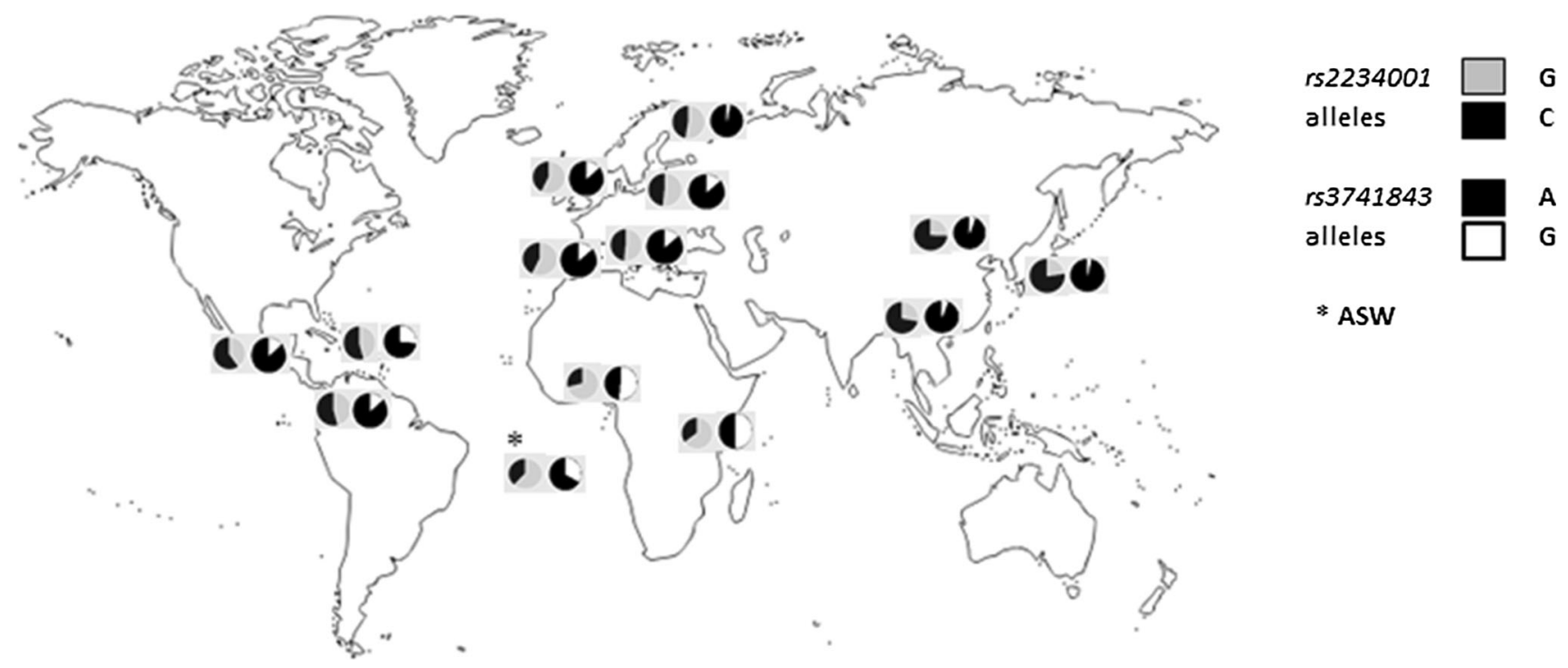

Fig. 5 Worldwide distribution of the studied TAS2R4 rs2234001 and TAS2R14 rs3741843 SNPs

shown in Table 3, $19.62 \%$ of variation at rs3741843 was due to differences among population groups $(P=0.002)$, $1.32 \%$ was due to differences among populations within groups $(P<0.001)$, and $79.06 \%$ was accounted by differences among individuals within populations $(P<0.001)$. rs2234001 showed a similar pattern, with $11.75 \%$ of variation which was attributed to differences among groups $(P<0.001)$, with the remaining $88.25 \%$ accounting only for differences observable within populations $(P=0.006)$.

\section{Genetic differentiation analyses}

Genome-wide pairwise Wright's $F_{S T}$ was calculated as a measure of genetic differentiation on 1000 Genomes populations and continental groups, and a set of relevant percentiles was extracted (Online Resource 5). F $_{\text {ST }}$ obtained for rs3741843 and rs2234001 fell within the top percentiles (0.95) of most of the continent-wide distributions and turned out to be outlier values also for some populationlevel comparison. 
Table 3 AMOVA performed on TAS2R14 rs3741843 and TAS2R4 rs2234001 SNPs

\begin{tabular}{lll}
\hline & $r s 3741843$ & $r s 2234001$ \\
\hline Among groups $^{\mathrm{a}}$ & $19.62 \%$ & $11.75 \%$ \\
Among population with $^{\text {groups }}$ & $1.32 \%$ & 0 \\
Within populations $^{\mathrm{b}}$ & $79.06 \%$ & \\
$\mathrm{~F}_{\mathrm{ct}}$ & 0.19619 & $88.25 \%$ \\
& $(P$ value 0.0019$)$ & $(P$ value $<0.001)$ \\
$\mathrm{F}_{\mathrm{sc}}$ & 0.01644 & 0 \\
& $(P$ value $<0.001)$ & \\
$\mathrm{F}_{\mathrm{st}}$ & 0.20940 & 0.1164 \\
& $(P$ value $<0.001)$ & $(P$ value 0.00587$)$
\end{tabular}

a Africans, Americans, Asian, Europeans

b YRI, LWK, ASW, CLM, MXL, PUR, CHB, JPT, CHS, CEU, FIN, GBR, IBS, TSI

\section{Admixture analysis}

The admixed nature of the American 1000 Genomes populations allowed us to test whether the studied SNPs could be differentially selected according to the ancestral components of these populations. The American, African and European genomic components of the 1000 Genomes American populations were extracted from the literature (1000 Genomes Project Consortium et al. 2012; Skotte et al. 2013) and used to infer the expected frequencies of the two SNPs, given the observed admixture. The African and European frequencies of the two SNPs were obtained from the relevant populations, while American frequencies were extracted from the Mexican one (MXL), after correcting for admixture. Such frequencies were then combined, according to their admixture proportions, to generate the expected frequencies of each of the American populations. Table 4 reports the observed and expected frequencies hence calculated, along with the observed excess. The difference between observed and expected frequencies is remarkable for rs3741843, where Colombians (CLM) show a defect of $22 \%$ and Puertoricans (PUR) show an excess of $61 \%$ on the expected frequencies.

\section{Discussion}

To our knowledge, this study represents the first attempt to explore genotype-phenotype association of stevioside bitter taste perception, as well its relationships with PROP phenotypes/genotypes. In fact, a previous study by Allen et al. (2013b) tested only for relationships between other steviol glycosides, RebA and RebD, and TAS2R SNPs. For this purpose, we have assayed three SNPs on TAS2R14 (rs11610105, rs3741843 and rs7138535), one on TAS2R4 (rs2234001) and three on TAS2R38 (rs10246939, rs1726866 and rs713598) genes for individual associations with stevioside perception phenotypes and for their relationships with PROP genotypes/phenotypes on a sample of 86 Italian subjects.

We confirmed the associations between PROP variants and PROP-taster status at both allelic and haplotypic levels. Surprisingly, compared to other published data reporting a correlation between PROP bitterness and intensity of different tastants, including sucrose, citric acid, sodium chloride and quinine (Hayes et al. 2008), the sweetness of aspartame (Duffy et al. 2006) and Acesulfame K (Allen et al. 2013a), as well as sweet foods, such as marshmallows and iced cakes (Lanier et al. 2005), we did not find any correlation between PROP bitterness and either stevioside sweetness or stevioside bitterness.

Stevioside perception showed great variability among the candidate subjects, with the majority of the individuals perceiving both a sweet and a bitter after-taste and some individuals being not able to perceive its bitterness or sweetness. Moreover, also bitterness intensity strongly varied across subjects. Investigating polymorphisms on TAS214 and TAS2R4 genes, which have been recently identified as the loci encoding for receptors that mediate the bitter off-taste of steviol glycosides in vitro (Hellfritsch et al. 2012), our results suggest that two SNPs (rs2234001 and rs3741843) contribute to the perceived bitterness of stevioside in humans. After phenotype identification, we showed that TAS2R4 rs2234001 varies among people who were able to perceive or not the bitter taste of stevioside. Specifically, our results demonstrate that the $G$ allele of
Table 4 Ancestral proportions in American 1000 Genomes populations and observed/ expected frequencies of TAS2R4 rs2234001 and TAS2R14 rs3741843 SNPs

a $\%$ of Native Americans in the Mexican population

\begin{tabular}{|c|c|c|c|c|c|c|c|c|c|}
\hline \multirow[t]{2}{*}{ Population } & \multicolumn{3}{|c|}{ Ancestral proportions } & \multicolumn{3}{|c|}{ rs 2234001} & \multicolumn{3}{|c|}{ rs3741843 } \\
\hline & $\%$ African & $\%$ NatAm & $\%$ European & Obs. & Exp. & $\%$ Excess & Obs. & Exp. & $\%$ Excess \\
\hline ASW & 0.79 & 0.02 & 0.19 & 0.39 & 0.37 & 4.65 & 0.38 & 0.40 & -6.06 \\
\hline CLM & 0.07 & 0.24 & 0.69 & 0.54 & 0.51 & 5.98 & 0.11 & 0.14 & -21.75 \\
\hline MXL & 0.02 & 0.60 & 0.38 & 0.60 & 0.60 & 0.00 & 0.11 & 0.11 & 0.00 \\
\hline PUR & 0.13 & 0.13 & 0.74 & 0.55 & 0.48 & 14.08 & 0.26 & 0.16 & 61.41 \\
\hline AFR & - & - & - & 0.34 & - & - & 0.48 & - & - \\
\hline EUR & - & - & - & 0.47 & - & - & 0.12 & - & - \\
\hline NATAM $(\mathrm{MXL})^{\mathrm{a}}$ & - & - & - & 0.69 & - & - & 0.08 & - & - \\
\hline
\end{tabular}


this locus was associated with capability to perceive bitter after-taste of stevioside, while the $\mathrm{C}$ allele was more abundant in subjects who could not perceive it. At the genotype level, the recessive homozygote status GG was associated with the ability to perceive the bitter taste of stevioside, while the dominant CC homozygote status was associated with the inability.

In addition, when comparing strong and low bitter stevioside perceptions, one SNP located on the TAS2R14 gene (rs3741843) showed significant frequency differences. In particular, its $\mathrm{G}$ allele was associated with a stronger bitter perception. In order to confirm this, we tried to verify whether perception of stevioside bitterness varied with the genotype and, as a matter of fact, the AA genotype turned out to be strongly correlated with lower values of bitter perception. Regarding PROP status, we did not find any relationships with neither stevioside perception nor the TAS2R4/14 studied SNPs, although minimal evidence that the rs3741843 AA genotype is predictive of PROP bitterness was observed.

While variation at rs 3741843 locus was not linked with any protein modification, we found that not only rs2234001 is a non-synonymous (albeit not deleterious) SNP, but that it is also in strong $\operatorname{LD}\left(r^{2}>0.9\right)$ with two other deleterious mutations in CEU (Northern Europeans from Utah). Both such polymorphisms (rs2227264 and rs2233998) were found to be associated with eating behavior (Dotson et al. 2010), while only rs 2227264 has been proved to be related to the perceived bitterness of the espresso coffee (Hayes et al. 2011), hence representing putative functional candidates for the reported genotype-phenotype association.

Patterns of worldwide variation of two SNPs related to stevioside perception, together with comparison of their pairwise population $F_{\text {st }}$ values with empirical genome wide distributions, highlighted great variability levels within human populations. In particular, rs2234001 showed a significant differentiation between African-Asian groups $(P=0.05)$, but not between Asian-European ones $(P=0.10)$, while rs3741843 was highly differentiated between African-Asian $(P=0.025)$ and African-European $(P=0.05)$ groups (Table S5). Furthermore, considering the $10 \mathrm{kbp}$ haplotypes centered on the rs 3741843 locus, the modal haplotype class broadly differed between African and European populations (Online Resource 6).

The strong differentiation between African and European groups for this latter SNP allowed us to detect a $61 \%$ excess of the African variant in Puertoricans, after accounting for their history of genetic admixture. The enrichment of the African component in Puertoricans may suggest the presence of some natural or social selective pressure acting on such variant after its arrival in their genic pool some 200 ya (Kidd et al. 2012). Such selective pressure could perhaps be related with cultural/dietary shifts caused by the introduction of African traditions at the time of their arrival in Puerto Rico. While these results might be affected by our choice of using the corrected Mexicans as proxy for the ancestral American components, we believe such choice to be conservative given that only two observed-expected pairs showed an excess higher than $20 \%$.

In conclusion, we found genotype-phenotype associations between stevioside perception and SNPs at the $T A S 2 R 4$ and TAS2R14 genes, which are significantly differentiated worldwide. This finding and the strong LD of one of these variants with deleterious mutations suggest a role played by natural selection in shaping the current patterns of variation observed in different human groups.

Acknowledgments We are very grateful to the "Slow Food Communities," Silvano Zaccone, Luisella Verderi and to all the people who agreed to participate to this study. This work was supported by the Fondazione Del Monte "Taste Genetic Geography" Grant to DL and by The Wellcome Trust (098051) to LP.

Ethical standards Davide Risso, Gabriella Morini, Luca Pagani, Andrea Quagliariello, Cristina Giuliani, Sara De Fanti, Marco Sazzini, Donata Luiselli and Sergio Tofanelli declare that they have no conflict of interest. All procedures followed were in accordance with the ethical standards of the Ethics Committee of the University of Bologna and with the Helsinki Declaration of 1975, as revised in 2000. Informed consent was obtained from all subjects for being included in the study.

\section{References}

Allen AL, McGeary JE, Knopik VS, Hayes JE (2013a) Bitterness of the non-nutritive acesulfame potassium varies with polymorphisms in TAS2R9 and TAS2R31. Chem Senses 38(5):379-389

Allen AL, McGeary JE, Hayes J (2013b) Rebaudioside A and Rebaudioside D bitterness do not covary with Acesulfame-K bitterness or polymorphisms in TAS2R9 and TAS2R31. Chemosens Percept 6(3):109-117

Baranowski et al (2011) 6-n-propylthiouracil taster status not related to reported cruciferous vegetable intake among ethnically diverse children. Nutr Res 31(8):594-600

Barrett JC, Fry B, Malle J, Daly MJ (2005) Haploview: analysis and visualization of $\mathrm{LD}$ and haplotype maps. Bioinformatics 21(2):263-265

Bufe B, Breslin PA, Kuhn C, Reed DR, Tharp CD, Slack JP, Kim UK, Drayna D, Meyerhof W (2005) The molecular basis of individual differences in phenylthiocarbamide and propylthiouracil bitterness perception. Curr Biol 15:322-327

Campbell MC, Ranciaro A et al (2013) Origin and differential selection of allelic variation at TAS2R16 associated with salicin bitter taste sensitivity in Africa. Mol Biol Evol 31(2):288-302

Caremoli F (2011) Bitter taste perception: a preliminary study of the effects on food preferences and potential applications in human nutrition. Master's Thesis in Food Science and Human Nutrition, University of Milan, Italy

Crammer B, Ikan R (1987) Progress in the chemistry and properties of the rebaudiosides. In: Grenby TH (ed) Developments in sweeteners. Elsevier Applied Science, London, pp 45-64 
Dotson C, Shaw H, Mitchell B, Munger S, Steinle N (2010) Variation in the gene TAS2R38 is associated with the eating behavior disinhibition in old order Amish women. Appetite 54(1):93-99

DuBois G, Prakash I (2012) Non-caloric sweeteners, sweetness modulators, and sweetener enhancers. Ann Rev Food Sci Technol 3:353-380

DuBois GE, Walters DE, Schiffman SS, Warwick ZS, Booth BJ, Pecore SD, Gibes K, Carr BT, Brands LM (1991) Concentrationresponse relationships of sweeteners. A systematic study. In: Walters DE, Orthoefer F, DuBois GE (eds) Sweeteners: discovery, molecular design and chemoreception, American Chemical Society Symposium Series 450. ACS Books, Washington, pp 261-276

Duffy VB, Davidson AC, Kidd JR, Kidd KK, Speed WC, Pakstis AJ, Reed DR, Snyder DJ, Bartoshuk LM (2004) Bitter receptor gene (TAS2R38), 6-n-propylthiouracil (PROP) bitterness and alcohol intake.Alcohol Clin Exp Res 28(11):1629-1637

Duffy VB, Hayes JE, Dinehart ME (2006) Genetic differences in sweet taste perception. In: Spillane WJ (ed) Optimising the sweet taste in foods. Woodhead Publishing, Cambridge, pp 30-53

Excoffier L, Laval G, Schneider S (2007) Arlequin (version 3.0): an integrated software package for population genetics data analysis. Evol Bioinform Online 23(1):47-50

Fox AL (1932) The relationship between chemical constitution and taste. Proc Nat Acad Sci USA 18:115-120

Green BG, Shaffer GS, Gilmore MM (1993) Derivation and evaluation of a semantic scale of oral sensation magnitude with apparent ratio properties. Chem Senses 18(6):683-702

Hayes JE, Bartoshuk LM, Kidd JR, Duffy VB (2008) Supertasting and PROP bitterness depends on more than the TAS2R38 gene. Chem Senses 33(3):255-265

Hayes JE, Wallace MR, Knopik VS, Herbstman DM, Bartoshuk LM, Duffy VB (2011) Allelic variation in TAS2R bitter receptor genes associates with variation in sensations from and ingestive behaviors toward common bitter beverages in adults. Chem Senses 36(3):311-319

Hellfritsch C, Brockhoff A, Stähler F, Meyerhof W, Hofmann T (2012) Human psychometric and taste receptor responses to steviol glycosides. J Agric Food Chem 60:6782-6793

Kidd J, Gravel S, Byrnes J et al (2012) Population genetic inference from personal genome data: impact of ancestry and admixture on human genomic variation. Am J Hum Genet 91(4):660-671

Kim UK, Jorgenson E, Coon H, Leppert M, Risch N, Drayna D (2003) Positional cloning of the human quantitative trait locus underlying taste sensitivity to phenylthiocarbamide. Science 299(5610):1221-1225

Kinghorn AD (2002) The genus Stevia. Taylor \& Francis, London

Kinghorn AD, Soejarto DD (1986) Sweetening agents of plant origin. Crit Rev Plant Sci 4:79-120

Kumar P, Henikoff S, Ng P (2009) Predicting the effects of coding non-synonymous variants on protein function using the SIFT algorithm. Nat Protoc 4:1073-1081

Lanier SA, Hayes JE, Duffy VB (2005) Sweet and bitter tastes of alcoholic beverages mediate alcohol intake in of-age undergraduates. Physiol Behav 83(5):821-831
Meyerhof W, Batram C, Kuhn C, Brockhoff A, Chudoba E, Bufe B, Appendino G, Behrens M (2010) The molecular receptive ranges of human TAS2R bitter taste receptors. Chem Senses 35(2): 157-170

Prakash I, DuBois GE, Clos JF, Wilkens KL, Fosdick LE (2008) Development of rebiana, a natural non-caloric sweetener. Food Chem Toxicol 46(Suppl. 7S):75-82

Pronin AN, Xu H, Tang H, Zhang L, Li Q, Li X (2007) Specific alleles of bitter receptor genes influence human sensitivity to the bitterness of aloin and saccharin. Curr Biol 17:1403-1408

Purcell S, Neale B, Todd-Brown K, Thomas L, Ferreira MA, Bender D, Maller J, Sklar P, de Bakker PI, Daly MJ, Sham PC (2007) PLINK: a tool set for whole-genome association and populationbased linkage analyses. Am J Hum Genet 81(3):559-575

Reed DR, Zhu G, Breslin PA, Duke FF, Henders AK, Campbell MJ, Montgomery GW, Medland SE, Martin NG, Wright MJ (2010) The perception of quinine taste intensity is associated with common genetic variants in a bitter receptor cluster on chromosome 12. Hum Mol Genet 19(21):4278-4285

Reimann F, Tolhurst G, Gribble FM (2012) G-protein-coupled receptors in intestinal chemosensation. Cell Metab 15:421-431

Roudnitzky N, Bufe B, Thalmann S, Kuhn C, Gunn H, Xing C, Crider B, Behrens M, Meyerhof W, Wooding S (2011) Genomic, genetic and functional dissection of bitter taste responses to artificial sweeteners. Hum Mol Genet 20(17):3437-3449

Simons CT, Adam C, LeCourt G, Crawford c, Ward C, Meyerhof W, Slack JP (2008) The "bitter-sweet" truth of artificial sweeteners. In: DuBois G, Weerasinghe DK (eds) Sweetness and sweeteners. Biology, chemistry, and psychophysics. American Chemical Society, Washington, pp 335-354

Skotte L, Korneliussen TS, Albrechtsen A (2013) estimating individual admixture proportions from next generation sequencing data. Genetics 195(3):693-702

Stephens M, Smith NJ, Donnelly P (2001) A new statistical method for haplotype reconstruction from population data. Am J Hum Genet 68(4):978-989

Tepper BJ (2008) Nutritional implications of genetic taste variation: the role of PROP sensitivity and other taste phenotypes. Annu Rev Nutr 28:367-388

The 1000 Genomes Project Consortium (2012) An integrated map of genetic variation from 1,092 human genomes. Nature 491:56-65

Vega-Gálvez A, Lemus-Mondaca R, Zura-Bravo L, Ah-Hen K (2012) Stevia rebaudiana Bertoni, source of a high-potency natural sweetener: a comprehensive review on the biochemical, nutritional and functional aspects. Food Chem 132:1121-1132

Wooding S, Gunn H, Ramos P, Thalmann S, Xing C, Meyerhof W (2010) Genetics and bitter taste responses to goitrin, a plant toxin found in vegetables. Chem Senses 35(8):685-692

Yadav KS, Guleria P (2012) Steviol glycosides from Stevia: biosynthesis pathway review and their application in foods and medicine. Crit Rev Food Sci Nutr 52:988-998

Zhao L, Kirkmeyera S, Tepper B (2003) A paper screening test to assess genetic taste sensitivity to 6-n-propylthiouracil. Physiol Behav 78:625-633 